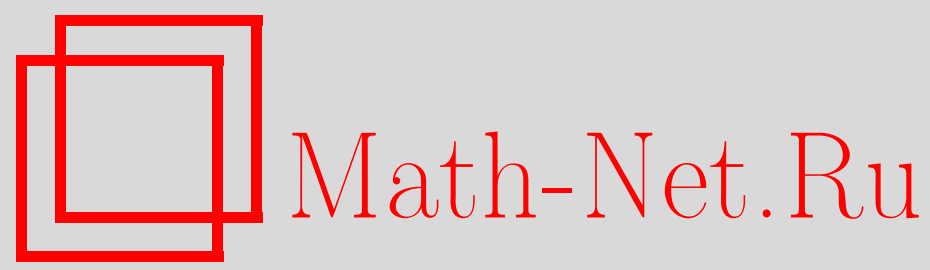

T. С. Рыбникова, О бесконечных системах линейных автономных и неавтономных стохастических уравнений, $\mathrm{Ma-}$ тем. заметки, 2002, том 71, выпуск 6, 890-901

DOI: https://doi.org/10.4213/mzm393

Использование Общероссийского математического портала Math-Net.Ru подразумевает, что вы прочитали и согласны с пользовательским соглашением http://www . mathnet.ru/rus/agreement

Параметры загрузки:

IP: 54.174 .149 .18

26 апреля 2023 г., $11: 26: 01$

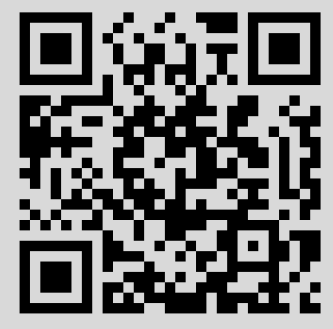




\section{О БЕСКОНЕЧНЫХ СИСТЕМАХ ЛИНЕЙНЫХ АВТОНОМНЫХ И НЕАВТОНОМНЫХ СТОХАСТИЧЕСКИХ УРАВНЕНИЙ}

\section{Т. С. Рыбникова}

Изучается разрешимость автономных и неавтономных линейных стохастических дифференциальных уравнений в пространстве $\mathbb{R}^{\infty}$. Доказьвается существование сильного непрерывного (непрерьвного в среднем степени $p$ ) решения автономного уравнения с непрерывной (соответственно непрерывной в среднем степени $p$ ) правой частью. Получены условия единственности решения. Приведен пример, показывающий, что обыкновенное и стохастическое линейные неавтономные уравнения с одинаковым оператором в $\mathbb{R}^{\infty}$ не всегда разрешимы. Для неавтономных стохастических уравнений найдены условия существования и единственности.

Библиография: 9 названий.

1. Введение. В работе рассматриваются бесконечные системы линейных стохастических дифференциальных уравнений вида

$$
d X_{n}(t)=d W_{n}(t)+\sum_{k=1}^{m_{n}} a_{n k}(t) X_{k}(t) d t
$$

Такие системы появляются во многих приложениях. Их можно трактовать как линейные стохастические дифференциальные уравнения в пространстве $\mathbb{R}^{\infty}$ всех последовательностей. В настоящей работе исследуется вопрос существования сильных решений автономных и неавтономных линейных стохастических дифференциальных уравнений в пространстве $\mathbb{R}^{\infty}$. Нетривиальность задачи связана с тем, что пространство $\mathbb{R}^{\infty}$ ненормируемо. В банаховьх пространствах обькновенное линейное дифференциальное уравнение и стохастическое линейное дифференциальное уравнение с непрерьвной (соответственно непрерьвной в среднем степени $p$ ) правой частью однозначно разрешимы. В ненормируемых локально выпуклых пространствах, даже в полных метрических пространствах ситуация иная (см. примеры в [1]): обыкновенное линейное дифференциальное уравнение и стохастическое линейное дифференциальное уравнение с непрерывным оператором не всегда разрешимы (см. также пример 4.6), с другой стороны, они могут иметь бесконечно много решений (см. пример 3.7).

К счастью, пространство $\mathbb{R}^{\infty}$ обладает особьми свойствами. Например, автономные системы линейных обыкновенных дифференциальных уравнений с непрерывньг оператором разрешимы при любом начальном условии (см. [2]). Это же верно и для стохастических линейных автономных уравнений с непрерьвной правой частью, что уже

Работа выполнена при частичной поддержке Российского фонда фундаментальных исследований, гранты № 00-15-99267 и № 01-01-00858, а также гранта DFG 436 RUS 113/343/0(R). 
было аннонсировано в [3] с наброском доказательства. Отметим, однако, что пространство $\mathbb{R}^{\infty}$ в этом отношении необычно: существуют пространства $Ф$ реше, в которых не каждое обыкновенное или стохастическое линейное дифференциальное уравнение с непрерьвньп оператором разрешимо (см. примеры в [1]). В п. 3 статьи мы приводим детали доказательства разрешимости линейного автономного стохастического уравнения в $\mathbb{R}^{\infty}$, не включенные в короткую заметку [3].

Другие результаты работы касаются неавтономных систем и взаимосвязи между детерминированным и стохастическим уравнениями с одним и тем же оператором. Приведен пример неразрешимого линейного неавтономного стохастического дифференциального уравнения в $\mathbb{R}^{\infty}$, получены условия существования и единственности решения для таких систем. Это обобщает результаты Герцога [4] для детерминированного случая.

2. Обозначения и терминология. Обозначим через $\mathbb{R}^{\infty}$ пространство всех действительных последовательностей, наделенное топологией покоординатной сходимости. Обозначим через $\mathbb{R}_{0}^{\infty}$ сопряженное к нему пространство, т.е. пространство всех финитных последовательностей. Пусть $\mathbb{R}_{+}=[0 ;+\infty)$. Обозначим через $C(E, F)$ пространство непрерывных отображений топологического пространства $E$ в топологическое пространство $F$. Через $C[a ; b]$ обозначим банахово пространство непрерывных вещественных функций на $[a ; b]$ с нормой $\|f\|_{C}=\max _{t \in[a ; b]}|f(t)|$. Для локально выпуклого пространства $E$ через $E^{*}$ обозначается его сопряженное, а через $\mathscr{L}(E)$ - пространство непрерьвных линейных операторов в $E$. Минимум из двух действительных чисел $a$ и $b$ обозначим через $a \wedge b$. Пусть $(\Omega, \mathscr{F}, P)$ - вероятностное пространство. Случайный процесс $\xi(t, \omega), t \in \mathbb{R}_{+}$, на $(\Omega, \mathscr{F}, P)$ со значениями в топологическом пространстве называется непрерывным, если почти все его траектории $\xi(\cdot, \omega)$ непрерывны. Отображение $A: \mathbb{R}_{+} \rightarrow \mathscr{L}\left(\mathbb{R}^{\infty}\right)$ называется непрерывныл $⿻$, если образ $A(t) x$ непрерывно зависит от $t$ для любого $x$ из $\mathbb{R}^{\infty}$.

Пусть $\left\{\mathscr{F}_{t}, t \in \mathbb{R}_{+}\right\}$- неубывающее семейство $\sigma$-алгебр, т.е. фильтраиия на $\Omega$. Мы будем предполагать, что фильтрация $\mathscr{F}_{t}$ полна, т.е. $\mathscr{F}_{0}$ содержит все множества меры нуль. Напомним, что случайньй процесс $\xi(\cdot): \mathbb{R}_{+} \times \Omega \rightarrow \mathbb{R}$ назьвается $\mathscr{F}_{t}$-согласованным (или согласованным ), если при любом $t \in \mathbb{R}_{+}$функция $\omega \rightarrow \xi(t, \omega)$ является $\mathscr{F}_{t}$-измеримой. Случайньй процесс $\xi(\cdot, \cdot)=\left(\xi_{n}(\cdot, \cdot)\right)$, определенный на пространстве $\mathbb{R}_{+} \times \Omega$, со значениями в пространстве $\mathbb{R}^{\infty}$ назьвается согласованным, если все его координаты $\xi_{n}(\cdot, \cdot)$ согласованы. Предсказуемой $\sigma$-алгеброй $\mathscr{P}$ на $[0 ;+\infty) \times \Omega$ назьвается $\sigma$-алгебра, порожденная множествами вида

$$
[s ; t] \times F, \quad \text { где } 0 \leqslant s<t<\infty, \quad F \in \mathscr{F}_{s} .
$$

Сужение $\mathscr{P}_{\infty}$ на $[0 ; T] \times \Omega$ обозначается через $\mathscr{P}_{T}$. Случайный процесс $\xi(\cdot, \cdot)$ на $[0 ;$ $+\infty) \times \Omega($ соответственно на $[0 ; T] \times \Omega)$ со значениями в $\mathbb{R}^{1}$ называется предсказуемым, если он измерим относительно $\mathscr{P}_{\infty}$ (соответственно относительно $\left.\mathscr{P}_{T}\right)$. Случайньй процесс $\xi(\cdot, \cdot)$ на $[0 ;+\infty) \times \Omega($ соответственно на $[0 ; T] \times \Omega)$ со значениями в $\mathbb{R}^{\infty}$ называется предсказуемым, если все его координаты предсказуемы.

Предсказуемые процессы согласованны. Согласованные непрерывные скалярные процессы являются предсказуемыми (см. [5, раздел 3.3$])$.

Наряду с непрерывными случайными процессами мы будем рассматривать процессы $\xi(\cdot)=\left(\xi_{n}(\cdot)\right)_{n=1}^{\infty}$ со значениями в пространстве $\mathbb{R}^{\infty}$, которые являются непрерьвньми в среднем степени $p \geqslant 1$, т.е. $\mathrm{E}\left|\xi_{n}(t)-\xi_{n}(s)\right|^{p} \rightarrow 0$ при $t \rightarrow s$ при каждом $n \in \mathbb{N}$. Другими словами, отображение $[0 ; T] \rightarrow L^{p}(\Omega, \mathscr{F}, P)$, которое переводит $t \mapsto \xi_{n}(t, \cdot)$, непрерьвно при всех $n \in \mathbb{N}$. При обсуждении свойств в среднем степени $p$ непрерьвность, 
гладкость, интеграл и производная случайной функции будут пониматься в смысле сходимости в $L^{p}(\Omega, \mathscr{F}, P)$. Производная в среднем степени $p$ случайного процесса определяется как предел в $L^{p}$ величин $\left(\xi_{t+h}-\xi_{t}\right) / h$ при $h \rightarrow 0$, а интеграл - как предел интегральных сумм Римана, которьй существует для всех непрерывных в среднем степени $p \geqslant 1$ процессов. При этом если некоторая случайная функция $\zeta_{t}, t \in[a ; b]$, измерима и непрерьвна в среднем степени $p \geqslant 1$, то почти наверное $\left(\left(L^{p}\right) \int_{a}^{t} \zeta_{s} d s\right)(\omega)=\int_{a}^{t} \zeta_{s}(\omega) d s$, где слева стоит интеграл в среднем, а справа - интеграл, определенньй отдельно для каждой реализации (см. [6, п. 2.1.4]). Отсюда следует, что если $\zeta(\cdot)$ - согласованньй непрерьвньй в среднем степени $p \geqslant 1$ процесс, то процесс $\int_{0}^{t} \zeta_{s}(\omega) d s$ также согласован. Легко видеть, что правая часть указанного равенства $\mathscr{F}_{t}$-согласована, значит, то же верно и для левой части. Детали см. в [6, п. 2.1.4].

Пусть заданы $f \in C\left(\mathbb{R}, \mathbb{R}^{\infty}\right)$ и согласованный случайньй процесс $\xi(\cdot)$ на $(\Omega, \mathscr{F}, P)$, принимающий значения в $\mathbb{R}^{\infty}$. Напомним, что оператор $A$ принадлежит $\mathscr{L}\left(\mathbb{R}^{\infty}\right)$ тогда и только тогда, когда его матрица $A$ содержит в каждой строке лишш конечное число ненулевых элементов. Предположим, что задано непрерывное отображение $A: \mathbb{R}_{+} \rightarrow \mathscr{L}\left(\mathbb{R}^{\infty}\right)$.

ОПРЕДЕЛЕНИЕ 2.1. Пусть $\xi(t)=\left(\xi(t, \omega), \omega \in \Omega, t \in \mathbb{R}_{+}\right)$- непрерьвньй (соответственно непрерьвньй в среднем степени $p$ ) согласованный случайньй процесс со значениями в $\mathbb{R}^{\infty}$. Процесс $X(t)=\left(X(t, \omega), \omega \in \Omega, t \in \mathbb{R}_{+}\right)$со значениями в $\mathbb{R}^{\infty}$ назьвается сильным решением уравнения

$$
d X(t)=A(t) X(t) d t+f(t) d t+d \xi(t), \quad X(0, \omega)=X_{0}(\omega)
$$

если он согласован и удовлетворяет для почти всех $\omega$ (соответственно в среднем степени $p$ ) интегральному уравнению

$$
X(t, \omega)=X_{0}(\omega)+\int_{0}^{t} A(t) X(s, \omega) d s+\int_{0}^{t} f(s) d s+\xi(t, \omega)-\xi(0, \omega) \quad \forall t \geqslant 0
$$

понимаемому покоординатно.

В частности, необходимо, чтобы каждая из координат процесса $A X(s, \omega)$ (т.е. функции $\left.\sum_{i=1}^{\infty} a_{n i}(s) X_{i}(s, \omega)\right)$ была локально интегрируема по $s$ при почти всех $\omega$. Для непрерьвных в среднем степени $p$ процессов необходимо, чтобы $X_{0 i}(\omega) \in L^{p}(\Omega, \mathscr{F}, P)$. Уравнение (2.2) эквивалентно уравнению

$$
d X(t)=A X(t) d t+d \zeta(t), \quad X(0, \omega)=X_{0}(\omega)
$$

где $\zeta(t, \omega)=\int_{0}^{t} f(s) d s+\xi(t, \omega)$, однако в некоторых ситуациях удобно бывает использовать запись $(2.2)$, например, ког да процесс $\xi(\cdot, \cdot)$ винеровский.

Последовательность непрерьвных случайных процессов $u_{n}(t, \omega)$ будем называть $c x о-$ дящейся $\kappa$ процессу $u(t, \omega)$ по вероятности равномерно по $t \in[0 ; T]$, если $P\{\omega$ : $\left.\left\|u_{n}(\cdot, \omega)-u(\cdot, \omega)\right\|_{C}>a\right\} \rightarrow 0$ для любого $a>0$ при $n \rightarrow \infty$ (т.е. соответствующие $C[0 ; T]$-значные случайные векторы сходятся по вероятности).

3. Автономные системы. Доказательства следуюших двух лемм приведены в работе [3]. Лемма 3.2 обобщает результат [2], доказанный для детерминированного случая (т.е. для $\xi \equiv 0)$. В случае стохастических уравнений возникает ряд технических трудностей, связанных с измеримостью решения. 
Лемма 3.1. (i) Для любого непрерывного согласованного скалярного случайного прочесса $\xi(t, \omega):[0 ; T] \times \Omega \rightarrow \mathbb{R}$ существует последовательность согласованных случайных процессов с гладкими траекториями $u_{n}(t, \omega)$, сходящаяся $\kappa \xi_{t}$ по вероятности равномерно по $t$ из $[0 ; T]$, причем $u_{n}(0) \equiv \xi(0), u_{n}^{(k)}(0) \equiv 0$ при всех $k \in \mathbb{N}$.

(ii) Для любого непрерывного в среднем степени р согласованного скалярного случайного прочесса $\xi(t, \omega), t \in[0 ; T]$, существует последовательность гладких в среднем степени р согласованных случайных прочессов $u_{n}(t, \omega)$, сходящаяся $\kappa \xi_{t}$ по вероятности равномерно по $t$ из $[0 ; T]$, причем $u_{n}(0) \equiv \xi(0), u_{n}^{(k)}(0) \equiv 0$ при вcex $k \in \mathbb{N}$.

ЛЕмма 3.2. Уравнение, имеющее в координатной форме вид

$$
d X_{n}(t)=X_{n+1}(t) d t+d \xi_{n}(t)+f_{n}(t) d t, \quad X(0)=\xi(0)
$$

имеет сильное решение $X$ для произвольных $f \in C\left(\mathbb{R}_{+}, \mathbb{R}^{\infty}\right)$ и $\mathscr{F}_{t}$-согласованного непрерывного (соответственно непрерывного в среднем степени р) случайного прочесса $\xi(t), t \in \mathbb{R}_{+}$, причем $X$ - непрерывный (соответственно непрерывный в среднем степени р) прочесс.

Следуюший результат хорошо известен и доказьвается методом последовательных приближений. По поводу близких результатов см. [1, теорема 6.15], [7].

Лемма 3.3. Пусть $E-$ банахово пространство, заданы некоторые $A \in \mathscr{L}(E)$, $f \in C\left(\mathbb{R}_{+}, E\right)$ и $\xi(t), t \in \mathbb{R}_{+}$, - непрерывный (соответственно непрерывный в среднем степени $p$ ) согласованный случайный процесс со значениями в Е. Тогда уравнение

$$
d X(t)=A X(t) d t+f(t) d t+d \xi(t), \quad X(0)=\xi(0),
$$

имеет единственное сильное решение $X$ со значениями в $E$, причем решение $X$ непрерывно (соответственно непрерывно в среднем степени $p$ ).

В дальнейшем нам понадобится следующая лемма, которую можно усмотреть из рассуждений в работе [2]. Однако для приложения к стохастическому случаю необходима более детальная формулировка.

Лемма 3.4. Пусть оператор $A \in \mathscr{L}\left(\mathbb{R}^{\infty}\right)$. Тогда найдется такой оператор $C \in \mathscr{L}\left(\mathbb{R}_{0}^{\infty}\right)$ c $C^{\top} \in \mathscr{L}\left(\mathbb{R}^{\infty}\right)$, ито $C^{\top} A\left(C^{\top}\right)^{-1}$ имеет вид

$$
A^{\prime}=\left(\begin{array}{cccccc}
A_{1} & 0 & & & \ldots & 0 \\
B_{21} & A_{2} & 0 & & \ldots & 0 \\
B_{31} & B_{32} & A_{3} & 0 & \ldots & 0 \\
\ldots & \ldots & \ldots & \ldots & \ldots & \ldots
\end{array}\right)
$$

где $A_{i}$ являются либо бесконечномерными блоками вида

$$
A_{i}=\left(\begin{array}{ccccc}
0 & 1 & 0 & 0 & \ldots \\
0 & 0 & 1 & 0 & \ldots \\
0 & 0 & 0 & 1 & \ldots \\
\ldots & \ldots & \ldots & \ldots & \ldots
\end{array}\right)
$$

либо конечномерными блоками, а $B_{i j}-$ блоки соответствующей размерности. 
ДокАЗАТЕЛьСтво. Пусть задан стандартньй базис $\left\{e_{k}\right\}_{k=1}^{\infty}, e_{k}=(0, \ldots, 0,1,0, \ldots)$, в $\mathbb{R}_{0}^{\infty}$. Построим новый базис $v_{i}=C e_{i}, i \in \mathbb{N}$, подобрав оператор $C$ так, чтобы композиция $C^{\top} A\left(C^{\top}\right)^{-1}$ имела бы форму (3.2). Процесс перехода к новому базису аналогичен описанному в [2]. Он состоит из одного или нескольких (возможно, счетного числа) шагов. Мы будем обозначать через $v_{i}^{n}$ вектор, построенный под номером $i$ на $n$-ом шаге. Одновременно мы будем строить матрицу $A^{\prime}$. На $n$-м шаге мы строим блоки $A_{n}, B_{n, 1}, \ldots, B_{n, n-1}$. Мы будем использовать индукцию по номеру шага, при этом внутри каждого шага новые векторы будут выбираться также индуктивно.

Шаг 1. Положим $v_{1}^{1}=e_{1}$. Если $A^{\top} v_{1}^{1} \in \operatorname{Lin}\left\{v_{1}^{1}\right\}$, то $A_{1}=\alpha$, где $\alpha \in \mathbb{R}$ - коэффициент такой, что $A^{\top} v_{1}^{1}=\alpha v_{1}^{1}$. Тогда $M_{1}=\operatorname{Lin}\left\{v_{1}^{1}\right\}$ и шаг завершен. В противном случае $A^{\top} v_{1}^{1} \notin \operatorname{Lin}\left\{v_{1}^{1}\right\}$ и мы положим $v_{2}^{1}=A^{\top} v_{1}^{1}$. Напомним, что в каждой строке матрицы $A$ содержится только конечное число ненулевых элементов. Следовательно, столбцы матрицы $A^{\top}$ имеют конечную длину и $v_{2}^{1} \in \mathbb{R}_{0}^{\infty}$. В этом случае шаг продолжается.

Опишем индуктивно его продолжение. Пусть шаг не завершен, и мы уже построили векторы $v_{i}^{1} \in \mathbb{R}_{0}^{\infty}$ для $i \leqslant j$. Обозначим $M=\operatorname{Lin}\left(v_{1}^{1}, \ldots, v_{j}^{1}\right)$.

Если $A^{\top} v_{j}^{1} \notin M$, то положим $v_{j+1}^{1}=A^{\top} v_{j}^{1}$. Вектор $v_{j}^{1} \in \mathbb{R}_{0}^{\infty}$, столбцы матрицы $A^{\top}$ также имеют конечное число элементов, поэтому $v_{j+1}^{1} \in \mathbb{R}_{0}^{\infty}$. Мы получили еще одну строку блока $A_{1}$ и матрицы $A^{\prime}$ : это строка номер $j$, в которой на $(j+1)$-м месте стоит единища, остальные элементы - нули. В этом случае шаг продолжается, и мы снова приходим к ситуации, описанной в предыдущем абзаце.

Если же $A^{\top} v_{1}^{j} \in M$, то $j$-й строкой блока $A_{1}$ и матрицы $A^{\prime}$ станут коэффициенты разложения этого вектора по базису $\left(v_{1}^{1}, \ldots, v_{j}^{1}\right)$, т.е. такие $\left(\alpha_{1}, \ldots, \alpha_{j}\right)$, что $A^{\top} v_{j}^{1}=$ $\sum_{i=1}^{j} \alpha_{i} v_{i}^{1}$. На этом шаг завершается.

Если $\operatorname{Lin}\left\{v_{i}^{1}\right\}=\mathbb{R}_{0}^{\infty}$, то процесс выбора нового базиса $\mathbb{R}_{0}^{\infty}$ заканчивается. В противном случае мы переходим к выполнению нового шага.

Опишем шаг с номером $n$. По индуктивному предположению заданы подпространства $M_{j}=\operatorname{Lin}\left\{v_{i}^{j}\right\}$ для $1 \leqslant j \leqslant n-1$, причем векторы $v_{i}^{j}$ являются линейно независимьми и число векторов, определенных при каждом $j$ (оно может оказаться и бесконечным), зависит от $j$. Обозначим $L_{n-1}=\operatorname{Lin}\left\{M_{1} \ldots M_{n-1}\right\}$. По индуктивному предположению будем считать, что векторы $e_{1}, \ldots, e_{n-1} \in L_{n-1}$ и блоки $A_{j}$ и $B_{j i}$ уже построены для $j \leqslant n-1, i<j$.

Шаг $n$. Положим $v_{1}^{n}$ равньм $e_{m}$, где $m=\min \left\{k: e_{k} \notin L_{n-1}\right\}$. Теперь будем строить векторы $v_{j}^{n}$ индукцией по $j$. Зафиксируем $j \in \mathbb{N}$. Предположим, что уже построены векторы $v_{i}^{n} \in \mathbb{R}^{\infty}$ при $i \leqslant j$ и задано подпространство $M_{n}=\operatorname{Lin}\left\{v_{i}^{n},\right\}, i \leqslant j$.

Если $A^{\top} v_{j}^{n} \notin M=\operatorname{Lin}\left(L_{n-1} \cup\left\{v_{1}^{n}, \ldots, v_{j}^{n}\right\}\right)$, то определим следующий вектор как $v_{j+1}^{n}=A^{\top} v_{j}^{n}$. По указанным вьше причинам $v_{j+1}^{n} \in \mathbb{R}_{0}^{\infty}$. Таким образом, построена новая строка матрищы $A_{n}$. Это строка номер $j$, на $(j+1)$-м месте которой стоит единища, остальные ее элементы - нули. Эта строка с остальными нулевыми элементами составит новую строку матрицы $A^{\prime}$. Все элементы $j$-й строки блоков $B_{n, 1}, \ldots, B_{n, n-1}$ будут нулями. В этом случае шаг продолжается, и мы возвращаемся к альтернативе, появившейся в начале шага $n$.

Если же $A^{\top} v_{j}^{n} \in M$, то следующей строкой матрицы $A^{\prime}$ будет вектор

$$
\left(\beta_{11}, \ldots, \beta_{i j}, \ldots, \alpha_{1}, \ldots, \alpha_{j}\right),
$$

определенный равенством

$$
A^{\top} v_{j}^{n}=\sum_{l=1}^{n-1} \sum_{i} \beta_{l i} v_{i}^{l}+\sum_{i=1}^{j} \alpha_{i} v_{i}^{n} .
$$


Отметим, что первая сумма - это проекция вектора $A^{\top} v_{j}^{n}$ на подпространство $L_{n-1}$, вторая сумма - это проекция того же вектора на $M_{n}=\operatorname{Lin}\left(v_{1}^{n}, \ldots, v_{j}^{n}\right)$. Тогда $j$-й строкой блока $A_{n}$ станет $\left(\alpha_{1}, \ldots, \alpha_{j}\right)$, в то время как $j$-й строкой $B_{n, k}, 1 \leqslant k \leqslant n-1$, будет $\left(\beta_{k 1}, \ldots, \beta_{k l}, \ldots\right), l \in\left\{i, e_{i} \in M_{k}\right\}$. На этом шаг завершается.

Если $\operatorname{Lin}\left\{M_{1}, \ldots, M_{n}\right\}=\mathbb{R}_{0}^{\infty}$, то процесс выбора нового базиса $\mathbb{R}_{0}^{\infty}$ завершен. Если же это не так, то мы переходим к выполнению следующего шага.

Описанньй вьше процесс состоит из конечного или бесконечного числа шагов. На выходе мы имеем систему векторов $H=\left\{v_{k}^{n}\right\}$, которая является алгебраическим базисом в пространстве $\mathbb{R}_{0}^{\infty}$. Действительно, по построению множество $H$ линейно независимо и его оболочка содержит все $e_{m}, m \in \mathbb{N}$. Матрица $C$, матрица перехода к новому базису, определена так, что композиция $C^{\top} A\left(C^{\top}\right)^{-1}$ имеет форму (3.2). Лемма доказана.

ТЕОРема 3.5. Уравнение

$$
d X(t)=A X(t) d t+f(t) d t+d \xi(t), \quad X(0)=X_{0},
$$

имеет сильное решение при любых $A \in \mathscr{L}\left(\mathbb{R}^{\infty}\right), f \in C\left(\mathbb{R}_{+}, \mathbb{R}^{\infty}\right)$ и для любого $\mathscr{F}_{t}$-согласованного непрерывного (соответственно непрерывного в среднем степени $p$ ) случайного процесса $\xi$ со значениями в пространстве $\mathbb{R}^{\infty}$. Причем проиесс $X(t, \omega)$ непрерывный (соответственно непрерывный в среднем степени $p$ ).

ДокАЗАТЕЛЬСтво. Рассмотрим уравнение

$$
d Y(t)=A^{\prime} Y(t) d t+g(t) d t+d \zeta(t),
$$

полученное из (3.3) линейньм преобразованием координат с матрищей $C^{\top}$, построенной в лемме 3.4. В (3.4) матрица $A^{\prime}$ имеет форму (3.2). Процесс $\xi(\cdot)$ является $\mathscr{F}_{t}$-согласованньп и непрерывным (соответственно непрерывным в степени $p$ ), поэтому $\zeta(\cdot)$ также обладает этими свойствами.

Почему система (3.4) разрешима? Пусть $A_{i}$ действуют в подпространствах $M_{i}$. Вопервых, сужение системы уравнений (3.4) на подпространство $M_{1}$ имеет вид

$$
d Y^{1}(t)=A_{1} Y^{1}(t) d t+g^{1}(t) d t+d \zeta^{1}(t)
$$

где $g^{1}(t)$ и $\zeta^{1}(t)$ - это проекции $g(t)$ и $\zeta(t)$ соответственно на подпространство $M_{1}$. Отметим, что система (3.5) либо является конечномерной, либо удовлетворяет условиям леммы 3.2. В обоих случаях система имеет сильное решение $Y^{1}(\cdot)$. Зная координаты $Y_{i}^{1}$ решения на подпространстве $M_{1}$, мы можем подставить их в уравнение (3.4). Теперь в уравнении (3.4) элементы всех блоков $B_{i 1}$, расположенных под $A_{1}$, умножаются на уже известные функции $Y_{i}^{1}$. Мы можем найти решение уравнения на подпространстве $M_{2}$. В этом случае мы приходим к системе

$$
d Y^{2}(t)=A_{2} Y^{2}(t) d t+g^{2}(t) d t+d \zeta^{2}(t)+B_{21} Y^{1}(t) d t
$$

где $g^{2}(t)$ и $\zeta^{2}(t)$ - это сужение соответственно $g(t)$ и $\zeta(t)$ на подпространство $M_{2}$. Система уравнений (3.6) разрешима по тем же причинам, что и система (3.5). Теперь, рассуждая по индукции, предположим, что нам известны сужения решения на подпространства $\left\{M_{1}, \ldots, M_{n-1}\right\}$. Подставив уже известные нам координаты решения в уравнение (3.4), мы найдем координаты решения $Y_{i}^{n}$ на $M_{n}$. Продолжая действовать таким образом, мы найдем координаты решения уравнения на всех $M_{n}$ при таких $n$, что $A_{n}$ определены. Наконец, мы найдем решение уравнения (3.3), применив обратное преобразование, полагая $x=\left(C^{\top}\right)^{-1} y$. Теорема доказана. 
ЗАмЕчАниЕ 3.6. В предыдущем доказательстве использованы идеи работы [2] (где рассматриваются детерминированные системы с $\xi=0$ ) и работы [8] (где рассматриваются системы, в которых $\xi_{n}$ - независимые стандартные винеровские процессы). Основная сложность в стохастическом случае - получить согласованное решение. Легко видеть, что в общем случае уравнение (3.3) может иметь несогласованное решение и что согласованное решение (3.3) может быть не единственньгм.

Как уже отмечалось в [3], доказательство основной теоремы в [8] содержит пробел. Данное вьше рассуждение не устраняет этот пробел, а следует по иному пути, позволяющему рассматривать более общие процессы. Тем самьм, конечно, оказывается реабилитирован и результат из [8].

ПРИмеР 3.7. Рассмотрим уравнение

$$
x_{n}^{\prime}(t)=x_{n+1}(t), \quad x_{n}(0)=z_{n}
$$

Легко видеть, что для каждого начального значения $z$ уравнение имеет бесконечно много решений.

Действительно, по теореме Бореля существует функция $\varphi$ класса $C^{\infty}$ такая, что $\varphi^{(n)}(0)=z_{n}$ для всех $n$. Функция $\varphi$ порождает решение

$$
x_{n}(t)=\varphi^{(n)}(t), \quad x_{n}(0)=z_{n} .
$$

Очевидно, что такая функция $\varphi$ не единственна, их бесконечно много и, следовательно, уравнение имеет бесконечно много решений.

Рассмотрим теперь уравнение (3.1). Прибавляя к решению уравнения (3.1), построенному в лемме 3.2 , произвольное решение однородного уравнения с нулевьм начальным условием, мы получаем новое решение уравнения (3.1). Однородное уравнение имеет бесконечно много решений, это верно и для уравнения (3.1).

Далее, пусть $W(\cdot, \cdot)$ - винеровский процесс на прямой и $x_{n}(t, \omega)=W(1, \omega) \cdot \varphi^{(n)}(t)$. Для того чтобы получить несогласованное решение $(3.1)$, прибавим $x(t, \omega)=\left(x_{n}(t\right.$, $\omega))_{n=1}^{\infty}$ к решению уравнения (3.1), построенному в лемме 3.2 .

ЗАмЕчАнИЕ 3.8. В условиях теоремы предположим, что процесс $\xi(t)$ непрерьвен. По условию теоремы процесс $\xi(t)$ согласован, следовательно, он предсказуем. Тогда решение $X(t)$ уравнения (3.3) также предсказуемо, поскольку процесс $X(t)$ является $\mathscr{F}_{t}$-согласованным и непрерывным.

ЗАмЕчАниЕ 3.9. (i) Из доказательства теоремы видно, что если процесс $\xi(t)$ гауссовский, то это же можно сказать и про построенное решение уравнения (3.4). Однако не каждое согласованное решение уравнения (3.4) будет гауссовским. Рассмотрим уравнение (3.1). Разность двух его решений с одинаковыми начальными условиями удовлетворяет (3.8) с нулевьги начальньгми условиями. Рассмотрим гладкую функцию $g(t)$, $t \in \mathbb{R}_{+}$, которая равна нулю на отрезке $[0 ; 1 / 2]$ и равна единице на $[1,+\infty)$. Пусть $\varphi(t, \omega)=g(t) \operatorname{sign}(\xi(1 / 2, \omega))$. Тогда $x_{n}(t)=\varphi^{(n)}(t)-$ согласованное негауссовское решение уравнения (3.8). Складывая данное решение однородного уравнения с решением (3.1), построенным в лемме 3.2 , получим сильное негауссовское решение уравнения (3.1).

(ii) Пусть процесс $\xi(t)$ в (3.4) имеет конечное математическое ожидание. Тогда если решение не единственно, найдутся сильные решения уравнения (3.4) с бесконечньпм 
математическим ожиданием, поскольку уравнение (3.8) с нулевыми начальными условиями имеет согласованное решение с бесконечньм математическим ожиданием. Им будет, например, решение (3.8), порожденное $\varphi(t, \omega)=g(t) \cdot \exp \left\{[\xi(1 / 2, \omega)]^{2}\right\}$, где функция $g(t)$ - такая же, как и в пункте $(\mathrm{i})$, а $\xi(\cdot)$ - винеровский процесс на прямой.

ЗАмЕчАниЕ 3.10. Следующие утверждения эквивалентны:

(a) уравнение (3.3) однозначно разрешимо;

(b) однородное уравнение $d X(t)=A X(t) d t, X(0)=0$, однозначно разрешимо;

(c) ряд

$$
\exp (t A)=\sum_{k=0}^{\infty} \frac{t^{k}}{k !} A^{k}
$$

сходится в $\mathscr{L}\left(\mathbb{R}^{\infty}\right)$ при всех $t \in \mathbb{R} ;$

(d) спектр $\sigma(A)$ не более чем счетен.

Утверждение (a) равносильно (b). Действительно, для любого $\omega$ разность двух решений уравнения (3.3) с одинаковыми начальными условиями удовлетворяет уравнению $d X(t)=A X(t) d t$ с нулевым начальньм условием. Утверждения (b) и (c) эквивалентны (см. [1, пример 4.24]). Наконец, равносильность утверждений (c) и (d) доказана в работе [9].

4. Неавтономные системы. В данном пункте рассматриваются неавтономные системы линейных дифференциальных уравнений. Приводится пример, показьвающий, что такие системы могут быть неразрешимы, в то время как автономные системы имеют решение при любом начальном значении.

Лемма 4.1. Пусть $A(\cdot)=\left(a_{i j}(\cdot)\right):[a ; b] \rightarrow \mathscr{L}\left(\mathbb{R}^{\infty}\right)$ - непрерывное отображение. Тогда для любого номера строки $i$ найдется номер $N(i)$, начиная с которого $a_{i j}(t) \equiv 0 н а[a ; b]$.

ДокАЗАтЕЛЬСтво. Предположим противное. Зафиксируем номер строки $k \in \mathbb{N}$. Пусть найдется бесконечная последовательность индексов $\left\{i_{n}\right\}, n \in \mathbb{N}$, такая, что элементы

$$
a_{k i_{1}}(\cdot), \ldots, a_{k i_{n}}(\cdot), \ldots
$$

$k$-й строки матрицы $A(\cdot)$ не обращаются тождественно в нуль. Рассмотрим семейство функционалов $\left\{a_{k}(t)\right\}$ на $\mathbb{R}^{\infty}$, где $a_{k}(t)=\left(a_{k 1}(t), \ldots, a_{k n}(t), \ldots\right), t \in[a ; b], k \in \mathbb{N}$. Это семейство равномерно ограничено на каждом $x \in \mathbb{R}^{\infty}$, поскольку отображение $A(\cdot)$ непрерьвно на $[a ; b]$, т.е. отображение $(A(\cdot) x)_{k}=\sum_{i=1}^{\infty} a_{k i}(\cdot) x_{i}$ непрерьвно по $t \in[a ; b]$ на каждом элементе $x \in \mathbb{R}^{\infty}$. Отсюда по теореме Банаха-Штейнгауза следует, что семейство $a_{k}(t)$ равномерно ограничено на каждом компактном множестве в пространстве $\mathbb{R}^{\infty}$. Однако это семейство функционалов не ограничено на множестве

$$
x_{i_{1}} \in\left[\frac{-1}{\left\|a_{k i_{1}}\right\|_{C}} ; \frac{1}{\left\|a_{k i_{1}}\right\|_{C}}\right], \quad \ldots, \quad x_{i_{n}} \in\left[\frac{-n}{\left\|a_{k i_{n}}\right\|_{C}} ; \frac{n}{\left\|a_{k i_{n}}\right\|_{C}}\right], \quad \ldots
$$

с нулевыми координатами $x_{j}$ при $j \notin\left\{i_{1}, i_{2}, \ldots, i_{n} \ldots\right\}$, которое является компактом в $\mathbb{R}^{\infty}$. Мы пришли к противоречию. Лемма доказана.

Поскольку обратное утверждение очевидно, мы получаем такой результат. 
СлЕДСтвиЕ 4.2. Отображсение $A(t)=\left(a_{i j}(t)\right):[a ; b] \rightarrow \mathscr{L}\left(\mathbb{R}^{\infty}\right)$ является непрерывным тогда и только тогда, когда все функиии $a_{i j}(\cdot)$ непрерывны и каждая строка содерэит лищь конечное число әлементов $a_{i j}(\cdot)$, не обращающихся тождественно в нуль на отрезке $[a ; b]$.

Пусть $Z(t)$ - матричнозначное отображение $[0 ; T] \rightarrow \mathscr{L}\left(\mathbb{R}_{0}^{\infty}\right) ;$ для $y \in \mathbb{R}_{0}^{\infty}$ положим

$$
U(Z, y)=\operatorname{lin}\left(\left\{Z\left(t_{1}\right) \cdots Z\left(t_{k}\right) y: t_{1}, \ldots, t_{k} \in[0 ; T], k \in \mathbb{N}\right\} \cup\{y\}\right)
$$

- наименъшее инвариантное относительно $Z(t), t \in[0 ; T]$, подпространство, содержащее $y$. Отображение $Z$ назьвается равномерно локально алгебраическим, если $\operatorname{dim} U(Z, y)<\infty$ для всех $y \in \mathbb{R}_{0}^{\infty}$.

В работе [4] получен следующий результат.

ПРЕДЛОЖЕНИЕ 4.3. Пусть отображение $A(t):[0 ; T] \rightarrow \mathscr{L}\left(\mathbb{R}^{\infty}\right)$ непрерывно, причем отображсние $A^{\top}(t):[0 ; T] \rightarrow \mathscr{L}\left(\mathbb{R}_{0}^{\infty}\right)$ является равномерно локально алгебраическим. Тогда уравнение

$$
y^{\prime}(t)=-A^{\top}(t) y(t), \quad y\left(t_{0}\right)=y_{0},
$$

однозначно разрешимо на $[0 ; T]$ при любом начальном значении $\left(t_{0}, y_{0}\right) \in[0 ; T] \times \mathbb{R}_{0}^{\infty}$. При любом начальном условии найдется дифферениируемая равномерно локально алгебраическая матричнозначная функиия $M(t):[0 ; T] \rightarrow \mathscr{L}\left(\mathbb{R}_{0}^{\infty}\right)$ такая, что $M(t)$ обратима на $\mathscr{L}\left(\mathbb{R}_{0}^{\infty}\right)$ при всех $t \in[0 ; T]$ и $y(t)=M(t) y_{0}, t \in[0 ; T]$, является решением уравнения (4.1). Более того, уравнение

$$
x^{\prime}(t)=A(t) x(t)+f(t), \quad x\left(t_{0}\right)=x_{0},
$$

однозначно разрешимо на $[0 ; T]$ при любом начальном значении $\left(t_{0}, x_{0}\right) \in[0 ; T] \times \mathbb{R}^{\infty}$ для всех $f \in C\left([0 ; T], \mathbb{R}^{\infty}\right)$. При любом начальном условии матричнозначная функиия $\Lambda(t):[0 ; T] \rightarrow \mathscr{L}\left(\mathbb{R}^{\infty}\right), \Lambda(t)=\left(M^{-1}\right)^{\top}(t)$, дифференичирума $и$

$$
x(t)=\Lambda(t) x_{0}+\int_{t_{0}}^{t} \Lambda(t) \Lambda^{(-1)}(s) f(s) d s, \quad t \in[0 ; T],
$$

является решением уравнения (4.2).

ЗАмЕЧАнИЕ 4.4. Отображение $x(t)=\Lambda(t) x_{0}$ является решением однородного уравнения $x^{\prime}(t)=A(t) x(t), x\left(t_{0}\right)=x_{0}$, соответствующего уравнению (4.2).

Перенесем сформулированный вьше результат на стохастические дифференциальные уравнения.

ПРЕДЛОЖЕНИЕ 4.5. Пусть отображение $A(t):[0 ; T] \rightarrow \mathscr{L}\left(\mathbb{R}^{\infty}\right)$ непрерывно, причем отображение $A^{\top}(t):[0 ; T] \rightarrow \mathscr{L}\left(\mathbb{R}_{0}^{\infty}\right)$ является равномерно локально алгебраическим. Тогда уравнение

$$
d X(t)=A(t) X(t) d t+d \xi(t), \quad X(0)=X_{0},
$$

имеет единственное сильное решение $X$ на отрезке $[0 ; T]$ для произвольного непрерывного (соответственно непрерывного в среднем степени р) согласованного случайного прочесса $\xi(t), t \in[0 ; T]$, со значениями в $\mathbb{R}^{\infty}$, причем решение $X$ непрерывно (соответственно непрерывно в среднем степени р) и задается формулой

$$
X(t, \omega)=\zeta(t, \omega)+\Lambda(t) \int_{0}^{t} \Lambda^{(-1)}(s) A(s) \zeta(s, \omega) d s, \quad t \in[0 ; T],
$$

əде $\zeta(t, \omega)=X_{0}(\omega)+\xi(t, \omega)-\xi(0, \omega)$. 
ДокАЗАТЕльСтво. Единственность сильного решения уравнения (4.3) вытекает из единственности решения однородного уравнения (4.2). Действительно, предположим, что $X_{1}(t, \omega)$ и $X_{2}(t, \omega)$ - два решения уравнения (4.3) с одинаковьпи начальными условиями. Тогда $X_{2}(t, \omega)-X_{1}(t, \omega)$ удовлетворяет однородному уравнению $(4.2)$ с нулевым начальным условием. По теореме 4.3 делаем вывод, что $X_{2}(t, \omega)-X_{1}(t, \omega)=0$, $t \in[0 ; T]$.

Формула (4.4) представляет из себя стандартное выражение для решения уравнения (4.3), и нам остается проверить, что правая часть равенства корректно определена и на самом деле задает решение. Здесь мы используем наши предположения об отображении $A(\cdot)$.

По теореме 4.3 матричнозначные отображения $\Lambda(\cdot)$ и $\Lambda^{(-1)}(\cdot)$ непрерьвны, отображение $A(\cdot)$ непрерьвно по предположению, следовательно, по лемме 4.1 интеграл в выражении (4.4) определен как в случае непрерывных процессов, так и в случае непрерывных в среднем степени $p$ процессов. Из (4.4) следует, что процесс $X(t, \omega)$ - согласованный.

Докажем, что процесс $X(\cdot, \cdot)$, определенньй равенством (4.4), является решением задачи (4.3). Для этого нам достаточно проверить, что

$$
\zeta(t, \omega)+\Lambda(t) \int_{0}^{t} \Lambda^{(-1)}(s) A(s) \zeta(s, \omega) d s=\zeta(t, \omega)+\int_{0}^{t} A(s) X(s) d s .
$$

Равенство верно для $t=0$. Вычитая $\xi(t, \omega)$ из обеих частей равенства (4.5) и дифференцируя (что обосновано леммой 4.1), имеем

$$
\Lambda^{\prime}(t) \int_{0}^{t} \Lambda^{(-1)}(s) A(s) \zeta(s, \omega) d s+\Lambda(t) \Lambda^{(-1)}(t) A(t) \zeta(t, \omega)=A(t) X(t) .
$$

Поскольку $\Lambda^{\prime}(t)=A(t) \Lambda(t)$ по построению, равенство (4.6) эквивалентно равенству

$$
A(t)\left(\Lambda(t) \int_{0}^{t} \Lambda^{(-1)}(s) A(s) \zeta(s, \omega) d s+\zeta(t, \omega)\right)=A(t) X(t) .
$$

Последнее выражение верно по (4.4). Следовательно, (4.4) действительно решение уравнения (4.3). Теорема доказана.

Следуюший пример является обобщением примера, построенного в работе [4] в случае детерминированных уравнений.

ПРимеР 4.6. Пусть задана последовательность $\left(f_{n}\right)_{n=1}^{\infty}$ непрерьвных функций на отрезке $[0 ; T]$ и винеровский процесс $W(\cdot, \cdot)$ на $[0, T] \times(\Omega, \mathscr{F}, P)$ со значениями в $\mathbb{R}^{1}$. Рассмотрим линейное однородное уравнение

$$
x_{n}(t)=x_{0 n}+\int_{0}^{t} f_{n}(s) x_{n+1}(s) d s, \quad n \in \mathbb{N},
$$

и линейное стохастическое уравнение

$$
\begin{aligned}
& \xi_{1}(t)=x_{01}+\int_{0}^{t} f_{1}(s) \xi_{2}(s) d s+W(t), \\
& \xi_{n}(t)=x_{0 n}+\int_{0}^{t} f_{n}(s) \xi_{n+1}(s) d s, \quad n \geqslant 2 .
\end{aligned}
$$

Тогда при некотором выборе последовательности $\left(f_{n}\right)_{n=1}^{\infty}$ уравнения $(4.7)$ и (4.8) оказьваются неразрешимыми для некоторьх начальных условий. 
ДокАЗАтЕльство. Рассмотрим сначала равномерно ограниченную последовательность $\left(f_{n}\right)_{n=1}^{\infty}$ непрерывных функций на $[0 ; T]$, принимающих неотрицательные значения и обладаюших свойством $\max \operatorname{supp} f_{n} \leqslant \min \operatorname{supp} f_{n+1}, n \in \mathbb{N}$. Тогда уравнение (4.7) однозначно разрешимо на отрезке $[0 ; T]$ и решение имеет вид

$$
x_{n}(t)=x_{0 n}+\int_{0}^{t} f_{n}(s) d s \cdot x_{0 n+1}, \quad t \in[0 ; T], \quad n \in \mathbb{N} .
$$

Полагая $\alpha_{n}=\int_{0}^{T} f_{n}(s) d s, k, n \in \mathbb{N}$, имеем

$$
x(T)=B x_{0}, \quad \text { где } B=\left(\begin{array}{ccccc}
1 & \alpha_{1} & 0 & 0 & \cdots \\
0 & 1 & \alpha_{2} & 0 & \cdots \\
0 & 0 & 1 & \alpha_{3} & \cdots \\
\vdots & \vdots & \vdots & \vdots &
\end{array}\right) \in L\left(\mathbb{R}^{\infty}\right) .
$$

Теперь пусть заданавозрастающая последовательность $\left(t_{k}\right)_{k=1}^{\infty}$ точек из [0;T] такая, что $t_{1}=0$ и $t_{k}<t_{k+1}, k \in \mathbb{N}$; пусть $t_{\infty}=\lim _{k \rightarrow \infty} t_{k}, t_{\infty}<T$. Рассмотрим равномерно ограниченную последовательность $\left(f_{n}\right)_{n=1}^{\infty}$ неотрицательных функций на $[0 ; T]$, у которых $\max \left(\left[t_{k}, t_{k+1}\right] \cap \operatorname{supp} f_{n}\right) \leqslant \min \left(\left[t_{k}, t_{k+1}\right] \cap \operatorname{supp} f_{n+1}\right), n, k \in \mathbb{N}$. Положим $\alpha_{k n}=\int_{t_{k}}^{t_{k+1}} f_{n}(s) d s, n, k \in \mathbb{N}$, и

$$
B_{k}=\left(\begin{array}{ccccc}
1 & \alpha_{k 1} & 0 & 0 & \cdots \\
0 & 1 & \alpha_{k 2} & 0 & \cdots \\
0 & 0 & 1 & \alpha_{k 3} & \cdots \\
\vdots & \vdots & \vdots & \vdots &
\end{array}\right) \in L\left(\mathbb{R}^{\infty}\right), \quad k \in \mathbb{N}
$$

Теперь задача (4.7) однозначно разрешима на отрезке $\left[0 ; t_{\infty}\right)$ и

$$
x\left(t_{k+1}\right)=B_{k} B_{k-1} \ldots B_{1} x_{0}, \quad k \in \mathbb{N} .
$$

Покажем, что существуют начальные значения $x_{0} \in \mathbb{R}^{\infty}$, при которых $x_{k 1}=x_{1}\left(t_{k}\right)$ $\rightarrow \infty$ при $k \rightarrow \infty$. Последовательность $f_{n}, n \in \mathbb{N}$, равномерно ограничена, поэтому $\alpha_{k n} \rightarrow 0$ при $k \rightarrow \infty$ при каждом $n$. Пусть $g(n)=\left(\alpha_{1, n-1} \alpha_{2, n-2} \cdots \alpha_{n-1,1}\right)^{(-1)}$. Тогда $g(n) \rightarrow \infty$ при $n \rightarrow \infty$. Предположим, что все координаты $x_{0, n}$ начального значения неотрицательны и сушествует бесконечно много $i \in \mathbb{N}$ таких, что $x_{0, n_{i}}>g\left(n_{i}\right)$. Тогда

$$
\begin{aligned}
x_{n_{i}-1,1}= & x_{0,1}+\alpha_{1,1} x_{0,2}+\alpha_{2,1}\left(x_{0,2}+\alpha_{1,2} x_{0,3}\right)+\cdots+\alpha_{n_{i}-1,1}\left(x_{0,2}+\cdots\right. \\
& \left.\quad+\alpha_{n_{i}-2,2} \alpha_{n_{i}-3,3} \cdots \alpha_{1, n_{i}-1} x_{0, n_{i}}\right) \\
= & x_{n_{i}-2,1}+\alpha_{n_{i}-1,1}\left(x_{0,2}+\cdots+\alpha_{n_{i}-2,2} \alpha_{n_{i}-3,3} \cdots \alpha_{1, n_{i}-1} x_{0, n_{i}}\right) \\
> & x_{n_{i}-2,1}+1
\end{aligned}
$$

и $x_{1}\left(t_{n_{i}}\right) \rightarrow \infty$. Следовательно, при таких начальных значениях уравнение (4.7) не имеет решения на отрезке $\left[0 ; t_{\infty}+\varepsilon\right]$ ни для какого $\varepsilon>0$.

Пусть $x(t)$ - построенное выше решение уравнения $(4.7)$ на $\left[0 ; t_{\infty}\right)$ с начальньм условием $x(0)=x_{0}$, для которого $x_{1}(t) \rightarrow \infty$ при $t \rightarrow t_{\infty}$. Пусть $\xi(t)-$ решение уравнения $(4.8)$ на отрезке [0; $\left.t_{\infty}\right)$ с тем же начальным условием. Тогда $\xi(t)$ задается равенствами $\xi_{n}(t)=x_{n}(t)$ при $n \geqslant 2$ и $\xi_{1}(t)=x_{1}(t)+W(t)$. Траектории $\xi_{1}(\cdot, \omega)$ неограничены при $t \rightarrow t_{\infty}$. Поэтому решение уравнения (4.8) не может быть продолжено на отрезок $\left[0 ; t_{\infty}+\varepsilon\right]$ ни при каком положительном $\varepsilon$ и данное уравнение не имеет решения на $[0 ; T]$ ни при каких начальных условиях. 
ЗАмЕчАниЕ 4.7. Вьше приведен пример неразрешимых обыкновенного линейного дифференциального уравнения и стохастического линейного дифференциального уравнения с одинаковыми операторами. Было бы интересно построить пример, когда одно из уравнений разрешимо, в то время как второе неразрешимо.

ЗАмЕчАниЕ 4.8. Полезно отметить, что все вьшеприведенные результаты верны для пространства $\mathbb{R}^{S}$, где $S$ - произвольное счетное множество. В этом легко убедиться, записав $s=\left\{s_{n}\right\}_{n=1}^{\infty}$ и использовав изоморфизм $\left(y_{n}\right)=\left(x_{s_{n}}\right)$ пространств $\mathbb{R}^{\infty}=\mathbb{R}^{\mathbb{N}}$ и $\mathbb{R}^{S}$.

Автор благодарна профессору В.И. Богачеву за внимание к работе, а также А. Пирковскому и О. Пугачеву за полезные обсуждения.

\section{СПИСОК ЦИТИРОВАННОЙ ЛИТЕРАТУРЫ}

[1] Bogachev V.I. Deterministic and stochastic differential equations in infinite-dimensional spaces // Acta Appl. Math. 1995. V. 40. № 1. P. 25-93.

[2] Шкарин С. А. Несколько результатов о разрешимости обыкновенных линейных дифференциальных уравнений в локально выпуклых пространствах // Матем. сб. 1990. Т. 181. № 9. C. $1183-1195$.

[3] Рыбникова Т. С. О разрешимости бесконечных систем линейных стохастических уравнений // Теория вероятностей и примен. 2000. Т. 45. № 3. С. 596-603.

[4] Herzog G. On linear time-dependent row-finite systems of differential equations. Travaux mathématiques Fescicule VIII // Séminaire de mathématique de Luxembourg. 1996. P. 167-176.

[5] Da Prato G., Zabczyk J. Stochastic Equations in Infinite Dimensions. Cambridge: Cambridge Univ. Press, 1992.

[6] Вентцель А. Д. Курс теории случайных процессов. М.: Физматлит, 1996.

[7] Sussman H. An interpretation of stochastic differential equations as ordinary differential equations which depend on the sample point // Bull. Amer. Math. Soc. 1977. V. 83. P. 296-298.

[8] Медницкий Ю. В. О существовании сильных решений линейных стохастических дифференциальных уравнений на $\mathbb{R}^{\infty} / /$ Теория вероятностей и примен. 1997. Т. 42. № 4. С. 826-831.

[9] Lemmert R., Weckbach $\ddot{A}$. Charakterisierungen zeilenendlicher Matrizen mit abzählbaren Spektrum // Math. Z. 1984. V. 188. №1. P. 119-129. 